\title{
HETEROGENEIDADE FLORÍSTICO-ESTRUTURAL DO COMPONENTE ARBÓREO EM UM SISTEMA DE FRAGMENTOS FLORESTAIS NO PLANALTO SUL CATARINENSE ${ }^{1}$
}

\author{
Marcelo Negrini², Pedro Higuchi ${ }^{3}$, Ana Carolina Silva ${ }^{4}$, Fábio Rodrigues Spiazzi ${ }^{5}$, Fernando Buzzi Junior ${ }^{6}$ e \\ Manoela Bez Vefago ${ }^{7}$
}

\begin{abstract}
RESUMO - Este trabalho teve como proposta analisar as variações espaciais, florísticas e estruturais do componente arbóreo de um sistema de fragmentos de Floresta Ombrófila Mista no Planalto catarinense. Para isso, foram alocadas 50 parcelas de $200 \mathrm{~m}^{2}(10 \times 20 \mathrm{~m})$ em quatro fragmentos, cujos indivíduos com diâmetro à altura do peito (DAP) $\geq 5 \mathrm{~cm}$ foram identificados e medidos. Foi calculado o Índice de Valor de Importância e realizada a comparação florístico-estrutural entre os fragmentos, por meio de Análise de Variância Multivariada Não Paramétrica (NPMANOVA). Espécies indicadoras de cada fragmento foram determinadas por meio da Análise de Espécies Indicadoras (ISA). Foram identificadas 73 espécies, sendo a de maior valor de importância Araucaria angustifolia (Bert.) Kuntze. Houve diferença na estrutura do componente arbóreo dos fragmentos $(p=0,001)$, sendo possível identificar espécies indicadoras para os fragmentos 1,2 e 3 . Este estudo demonstrou que fragmentos próximos entre si podem apresentar variações florístico-estruturais, que devem ser consideradas na definição de estratégias de manejo e conservação florestal.
\end{abstract}

Palavras-chave: Fragmentação florestal; Floresta com araucária; Análise multivariada.

\section{FLORISTIC-STRUCTURAL HETEROGENEITY OF THE TREE COMPONENT IN A SYSTEM OF FOREST FRAGMENTS IN THE SOUTHERNPLATEAU OF SANTA CATARINA, BRAZIL}

\begin{abstract}
The goal of this study was to evaluate the spatial, floristic and structural variations of the tree component of a fragments system of Araucaria Forest in the uplands of the state of Santa Catarina, Brazil. For this sake, within four fragments, 50 parcels of $200 \mathrm{~m}^{2}(10 \times 20 \mathrm{~m})$ were allocated; their individuals with diameter at breast height (DBH), measured at $1.30 \mathrm{~m}$ height, $\geq 5 \mathrm{~cm}$, were identified and measured. The Importance Value Index was determined and the floristic-structural comparison among fragments was analyzed, applying a non-parametric multivariate analysis of variance (NPMANOVA). Indicator species of each fragment was determined by Indicator Species Analysis (ISA). We found 73 species, being Araucaria angustifolia (Bert.) Kuntze the most important species. There was a difference in the tree component structure of the fragments ( $\mathrm{p}=0.001)$; it is possible to identify indicator species for fragments 1,2 and 3 . This study showed that fragments located close to each other may present floristic-structural variations, which should be considered during strategies definition for forest management and conservation.
\end{abstract}

Keywords: Forest fragmentation; Araucaria forest; Multivariate analysis.

\footnotetext{
${ }^{1}$ Recebido em 05.04.2013 aceito para publicação em 28.07.2014.

${ }^{2}$ FIT Manejo Florestal do Brasil, Brasil. E-mail: <engfmarcelonegrini@hotmail.com>.

${ }^{3}$ Departamento de Engenharia Florestal, Universidade do Estado de Santa Catarina, Centro Agroveterinário, Brasil. E-mail: $<$ higuchip@gmail.com>.

${ }^{4}$ Departamento de Engenharia Florestal, Universidade do Estado de Santa Catarina, Centro Agroveterinário, Brasil. E-mail: <carol_sil4@yahoo.com.br>.

${ }^{5}$ Instituto Federal de Santa Catarina, Brasil.. E-mail: <maxplus2007@yahoo.com.br>.

${ }^{6}$ Graduando em Engenharia Florestal pela Universidade do Estado de Santa Catarina, Centro Agroveterinário, Brasil. Email:<buzzifjr@hotmail.com>.

${ }^{7}$ Graduando em Engenharia Florestal pela Universidade do Estado de Santa Catarina, Centro Agroveterinário, Brasil. Email: <manuvefago@hotmail.com>.
} 


\section{INTRODUÇÃO}

A região do Planalto Sul catarinense é caracterizada por apresentar, como vegetação dominante, fragmentos naturais de Floresta Ombrófila Mista inseridos em uma matriz campestre. No entanto, devido a um processo de ocupação de terras, a vegetação natural tem sido substituída por áreas com usos alternativos da terra, como a urbanização, as atividades agrícolas e pecuárias e, mais recentemente, a silvicultura. Esse processo resultou em um mosaico de remanescentes vegetacionais, com diferentes áreas, formas e estágios de degradação.

Recentemente, estudos têm sido realizados com o objetivo de caracterizar a composição florística e estrutural em fragmentos florestais da região (KLAUBERG et al., 2010; NASCIMENTO et al., 2011; HIGUCHI et al., 2012a; NEGRINI et al., 2012; SILVA et al., 2012; HIGUCHI et al., 2013; SILVA et al., 2013). Esses estudos têm adotado uma escala espacial reduzida, investigando as variações que ocorrem no interior de cada fragmento. Entre os fatores ambientais determinantes nas variações que ocorrem no interior dos fragmentos na região, destacam-se os edáficos, os fisiográficos e os impactos ambientais (HIGUCHI et al., 2012a, 2013). No entanto, pouco se conhece a respeito das variações de parâmetros vegetacionais na escala de paisagem, ou seja, entre fragmentos. Nessa escala mais ampla, estudos em outras regiões têm demonstrado tanto a influência da estrutura da paisagem quanto do isolamento do fragmento (DISLICH et al., 2001), de fatores edáficos (MACHADO et al., 2004; SILVA et al., 2009) e de diferenças no estágio sucessional (DURIGAN et al., 2008) sobre a composição florística e estrutural da vegetação. Dessa forma, é presumível que fragmentos florestais, mesmo aqueles próximos geograficamente entre si, apresentem elevada variação estrutural e florística do componente arbóreo. Conhecer a organização espacial de comunidades arbóreas em remanescentes florestais, numa escala de paisagem, é fundamental para subsidiar estratégias de manejo florestal, visando à exploração sustentável e, ou, à conservação.

Considerando o contexto apresentado, este trabalho teve como objetivo testar a hipótese de que existem variações estruturais e florísticas em um sistema de quatro fragmentos de Floresta Ombrófila Mista, na região do Planalto Sul catarinense, refletindo uma possível heterogeneidade ambiental e diferentes históricos de perturbação na área.

\section{MATERIAL E MÉTODOS}

O estudo foi realizado em quatro fragmentos florestais na Bacia do Rio Caveiras, distanciados 29 a 50 m entre si (distância do vizinho mais próximo), localizados no Município de Lages, SC. A região possui clima temperado superúmido, sem estação seca (IBGE, 2002), com precipitação e temperatura médias anuais, respectivamente, de $1.682,80 \mathrm{~mm}$ e $15,9{ }^{\circ} \mathrm{C}$ (dados para o período de 1970 a 2010, obtidos da estação meteorológica de Lages, administrada pela EPAGRI/ CIRAM). A vegetação dos fragmentos pode ser classificada como Floresta Ombrófila Mista Montana (IBGE, 2012), sendo esses inseridos em uma matriz de campos naturais. Predominantemente, a região apresenta relevo com dissecação homogênea em forma de colina, com solos pertencentes às classes dos Cambissolos Húmicos e Terra Bruna Estruturada (EMBRAPA, 2004).

Para o levantamento florístico-estrutural, foram alocadas 50 parcelas de $200 \mathrm{~m}^{2}(10 \times 20 \mathrm{~m})$ nos quatro fragmentos estudados (15 parcelas nos fragmentos 1 e 4, respectivamente, com 8,4 e 25,2 ha e 10 parcelas nos fragmentos 2 e 3, com os respectivos 2,9 e 11,4 ha), totalizando 1 ha de área amostrada. As parcelas foram distribuídas nos fragmentos de forma estratificadasistemática, distantes $10 \mathrm{~m}$ entre si, de forma a amostrar adequadamente as suas variações ambientais, como borda e interior. Todos os indivíduos arbóreos e arborescentes vivos dentro das parcelas que apresentaram diâmetro à altura do peito (DAP, medido a $1,30 \mathrm{~m}$ de altura) igual ou superior a $5 \mathrm{~cm}$ foram identificados e tiveram seu DAP medido. Para complementar a lista florística, foram realizados caminhamentos aleatórios pelos fragmentos e identificadas espécies arbóreas não encontradas dentro das parcelas. As identificações foram realizadas por meio de literatura, e as espécies foram classificadas nas famílias, de acordo com o sistema APG III (ANGIOSPERM PHYLOGENY GROUP, 2009).

A suficiência da amostragem foi verificada pela curva de rarefação de espécies, elaborada no programa R (R DEVELOPMENT CORE TEAM, 2012), com a utilização do pacote Vegan (OKSANEN et al., 2012). Essa curva foi construída por meio do método de aleatorização, com 1.000 permutações. A distribuição dos valores de riqueza estimados para as unidades amostrais, por meio das permutações, foi inserida na curva de acumulação de espécies, utilizando gráficos do tipo "boxplot”. 
Foram calculados o índice de diversidade de Shannon ( $\left.\mathrm{H}^{\prime}\right)$ e a equabilidade de Pielou (J). A estrutura do componente arbóreo foi descrita a partir do cálculo, para cada espécie, do índice de valor de importância (IVI), calculado pela somatória das estimativas relativas de densidade, frequência e dominância (MUELLERDOMBOIS; ELLENBERG, 1974).

As parcelas foram ordenadas por meio de uma Análise de Correspondência Retificada (DCA), utilizando-se dados de abundância, com o propósito de verificar a organização estrutural da comunidade arbórea, em função dos fragmentos. Para isso, foram removidas as espécies com menos do que cinco indivíduos e, posteriormente, realizada a transformação $\log (\mathrm{x}+1)$, com o propósito de reduzir a assimetria entre as espécies abundantes e raras. Foi realizada uma análise de variância não paramétrica multivariada (NPMANOVA) para verificar a existência de diferenças significativas entre os fragmentos em relação à estrutura do componente arbóreo (ANDERSON, 2001). Para determinação das espécies indicadoras de cada um dos fragmentos, foi realizada uma análise de espécies indicadoras (ISA), sensu Dufrêne e Legendre (1997). As análises foram realizadas no programa $R(R$ DEVELOPMENT CORE TEAM, 2012), junto com os pacotes Vegan (OKSANEN et al., 2012) (DCA e NPMANOVA) e labdsv (ROBERTS, 2012) (ISA).

\section{RESULTADOS}

Nos quatro fragmentos, foram encontradas 73 espécies, distribuídas em 55 gêneros e 33 famílias botânicas, incluindo as espécies observadas somente no caminhamento (Tabela 1). Enquanto a maior parte das espécies (34) ocorreu em todos os fragmentos estudados, 16 espécies estiveram presentes em apenas um fragmento.

Tabela 1 - Famílias botânicas e número de indivíduos por espécies arbóreas encontradas em quatro fragmentos (F1, F2, F3 e F4) de Floresta Ombrófila Mista no Município de Lages, SC (*espécies encontradas somente na florística, isto é, fora das parcelas).

Table 1 - Botanical families and number of individuals per tree species found in four Araucaria Forest fragments (F1, F2, F3 and F4), in the municipality of Lages, SC (*species found only in floristics, i.e., outside the parcels).

\begin{tabular}{|c|c|c|c|c|c|}
\hline Famílias & Espécies & F1 & $\mathrm{F} 2$ & F3 & F4 \\
\hline \multirow[t]{3}{*}{ Anacardiaceae } & Lithraea brasiliensis Marchand & 9 & 13 & 4 & 14 \\
\hline & Schinus polygamus (Cav.) Cabrera & 1 & - & - & - \\
\hline & Schinus terebinthifolius Raddi & 8 & 7 & 5 & 3 \\
\hline Annonaceae & Annona rugulosa (Schltdl.) H. Rainer & 3 & 7 & 12 & 1 \\
\hline \multirow[t]{3}{*}{ Aquifoliaceae } & Ilex dumosa Reissek & 2 & - & - & - \\
\hline & Ilex microdonta Reissek & 3 & - & - & - \\
\hline & Ilex theezans Mart. ex Reissek & 13 & 5 & 5 & 2 \\
\hline Araliaceae & Oreopanax fulvus Marchal* & & & & \\
\hline Araucariaceae & Araucaria angustifolia (Bert.) Kuntze & 35 & 23 & 36 & 58 \\
\hline \multirow[t]{4}{*}{ Asteraceae } & Dasyphyllum spinescens (Less.) Cabrera & 1 & 1 & 9 & 3 \\
\hline & Dasyphyllum tomentosum (Spreng.) Cabrera & 2 & 7 & 1 & 3 \\
\hline & Gochnatia polymorpha (Less.) Cabrera & 7 & 2 & 2 & 1 \\
\hline & Vernonanthura discolor (Spreng.) H.Rob. & 5 & 10 & - & 4 \\
\hline Berberidaceae & Berberis laurina Billb.* & & & & \\
\hline Bignoniaceae & Jacaranda puberula Cham. & 37 & 6 & - & 5 \\
\hline Canelaceae & Cinnamodendron dinisii Schwacke & 10 & 21 & 18 & - \\
\hline Cannabaceae & Celtis brasiliensis (Gardner) Planch. & 2 & 1 & 1 & 3 \\
\hline Cardiopteridaceae & Citronella gongonha (Mart.) R.A.Howard & - & 2 & - & 1 \\
\hline Celastraceae & Maytenus dasyclada Mart. & 3 & - & - & - \\
\hline Clethraceae & Clethra scabra Pers. & 5 & 4 & 5 & 3 \\
\hline Cunoniaceae & Lamanonia ternata Vell. & - & - & 3 & 3 \\
\hline Dicksoniaceae & Dicksonia sellowiana Hook. & 5 & 4 & 3 & 9 \\
\hline
\end{tabular}


Tabela 1 - Cont.

Table 1 - Cont.

\begin{tabular}{|c|c|c|c|c|c|}
\hline Erythroxylaceae & Erythroxylum deciduum A.St.-Hil. & 2 & - & 1 & 2 \\
\hline \multirow{3}{*}{ Euphorbiaceae } & Sapium glandulosum (L.) Morong & 2 & 1 & 2 & 2 \\
\hline & Sebastiania brasiliensis Spreng. & 1 & - & 2 & 1 \\
\hline & Sebastiania commersoniana (Baill.) L. B. Sm. \& Downs & 16 & 36 & 12 & 15 \\
\hline \multirow[t]{3}{*}{ Fabaceae } & Dalbergia frutescens (Vell.) Britton & 8 & 30 & 7 & - \\
\hline & Inga lentiscifolia Benth. & - & - & 3 & - \\
\hline & Mimosa scabrella Benth. & - & 2 & 1 & - \\
\hline \multirow[t]{5}{*}{ Lauraceae } & Cinnamomum amoenum (Nees \& Mart.) Kosterm. & 4 & 2 & - & - \\
\hline & Ocotea puberula (Rich.) Nees & - & 3 & 1 & - \\
\hline & Ocotea pulchella Mart. & 5 & 6 & 3 & 2 \\
\hline & Nectandra megapotamica (Spreng.) Mez & - & - & - & 1 \\
\hline & Persea major L.E.Kopp & - & 2 & - & - \\
\hline Loganiaceae & Strychnos brasiliensis (Spreng.) Mart. & - & - & - & 1 \\
\hline \multirow[t]{17}{*}{ Myrtaceae } & Acca sellowiana (O.Berg) Burret & 1 & 3 & 1 & 4 \\
\hline & Blepharocalyx salicifolius (Kunth) O.Berg & 2 & 3 & 7 & 3 \\
\hline & Calyptranthes concinna DC. & 59 & 2 & 11 & 18 \\
\hline & Campomanesia xanthocarpa O. Berg & 5 & 7 & - & 2 \\
\hline & Eugenia pluriflora DC. & 13 & 2 & 1 & 9 \\
\hline & Eugenia pyriformis Cambess. & 1 & 3 & - & 1 \\
\hline & Eugenia uniflora L. & - & - & 1 & - \\
\hline & Myrceugenia euosma (O.Berg) D.Legrand & 1 & 1 & 4 & - \\
\hline & Myrceugenia myrcioides (Cambess.) O. Berg & 5 & 2 & 1 & 1 \\
\hline & Myrcia guianensis (Aubl.) DC. & 1 & - & 1 & - \\
\hline & Myrcia hatschbachii D.Legrand & - & 1 & - & - \\
\hline & Myrcia laruotteana Cambess. & 25 & 6 & 3 & 23 \\
\hline & Myrcia palustris DC. & 41 & 9 & 5 & 16 \\
\hline & Myrcia splendens (Sw.) DC. & 3 & - & - & - \\
\hline & Myrcianthes gigantea (D.Legrand) D.Legrand & 1 & - & - & - \\
\hline & Myrciaria delicatula (DC.) O.Berg & 28 & 7 & 5 & 10 \\
\hline & Myrrhinium atropurpureum Schott & 4 & 0 & 4 & 3 \\
\hline Podocarpaceae & Podocarpus lambertii Klotzsch & 14 & 9 & 63 & - \\
\hline \multirow[t]{2}{*}{ Primulaceae } & Myrsine coriacea (Sw.) Roem. \& Schult. & 11 & 5 & 15 & 5 \\
\hline & Myrsine umbellata Mart. & 1 & - & - & - \\
\hline Proteaceae & Roupala montana Aubl. & - & 5 & - & - \\
\hline Quillajaceae & Quillaja brasiliensis (A.St.-Hil. \& Tul.) Mart. & - & - & 1 & 1 \\
\hline Rhamnaceae & Scutia buxifolia Reissek & 10 & 14 & 25 & 18 \\
\hline Rosaceae & Prunus myrtifolia (L.) Urb. & 5 & 1 & 2 & 2 \\
\hline \multirow[t]{2}{*}{ Rutaceae } & Zanthoxylum kleinii (R.S.Cowan) P.G.Waterman & 6 & 5 & 15 & 2 \\
\hline & Zanthoxylum rhoifolium Lam. & 8 & 3 & 6 & 6 \\
\hline \multirow[t]{5}{*}{ Salicaceae } & Banara tomentosa Clos & 35 & 2 & 10 & 24 \\
\hline & Casearia decandra Jacq. & 20 & 11 & 13 & 8 \\
\hline & Casearia obliqua Spreng. & 3 & 8 & 1 & 1 \\
\hline & Xylosma ciliatifolia (Clos) Eichler & 1 & 10 & 1 & 1 \\
\hline & Xylosma tweediana (Clos) Eichler & - & 1 & 2 & - \\
\hline \multirow[t]{4}{*}{ Sapindaceae } & Allophylus edulis (A.St.-Hil., Cambess. \& A.Juss.) Radlk. & 1 & 8 & 2 & 11 \\
\hline & Allophylus guaraniticus (A.St.-Hil.) Radlk. & 2 & - & 5 & - \\
\hline & Cupania vernalis Cambess. & - & - & - & 1 \\
\hline & Matayba elaeagnoides Radlk. & 27 & 15 & 7 & 16 \\
\hline Solanaceae & Solanum sanctaecatharinae Dunal & - & 2 & - & - \\
\hline Symplocaceae & Symplocos uniflora (Pohl) Benth. & 3 & 5 & 7 & - \\
\hline Winteraceae & Drimys brasiliensis Miers & 27 & 3 & 6 & 10 \\
\hline
\end{tabular}

Revista Árvore, Viçosa-MG, v.38, n.5, p.779-786, 2014 
A curva de rarefação de espécie demonstrou tendência para a estabilização (Figura 1). Além disso, com a inclusão da última parcela, houve incremento médio menor do que 0,5\% no número de espécies, indicando que a intensidade amostral foi adequada para caracterizar a florística da área.

A diversidade ( $\mathrm{H}^{\prime}$ ) e a equabilidade $(\mathrm{J})$ dos fragmentos estudados foram, respectivamente, de 3,6 e 0,86. Myrtaceae destacou-se como a família de maior riqueza (17 espécies), seguida por Lauraceae (cinco espécies) e Salicaceae (cinco espécies).

A análise da estrutura horizontal, considerando todos os fragmentos, apontou Araucaria angustifolia (Bert.) Kuntze como a espécie com maior valor de importância (VI = 10,9\%), seguida por Matayba elaeagnoides Radlk. (VI = 6,6\%), Sebastiania commersoniana (Baill.) L. B. Sm. \& Downs (VI =4,6\%), Podocarpus lambertii Klotzsch (VI = 4,3\%) e Calyptranthes concinna DC. (VI = 4,1\%). Oito espécies ocorreram com apenas um indivíduo, totalizando 11,3\% do total de espécies amostradas nas parcelas (71).

A partir da ordenação das parcelas por meio da DCA, foi possível observar que a organização estrutural da comunidade arbórea não foi homogênea na área,

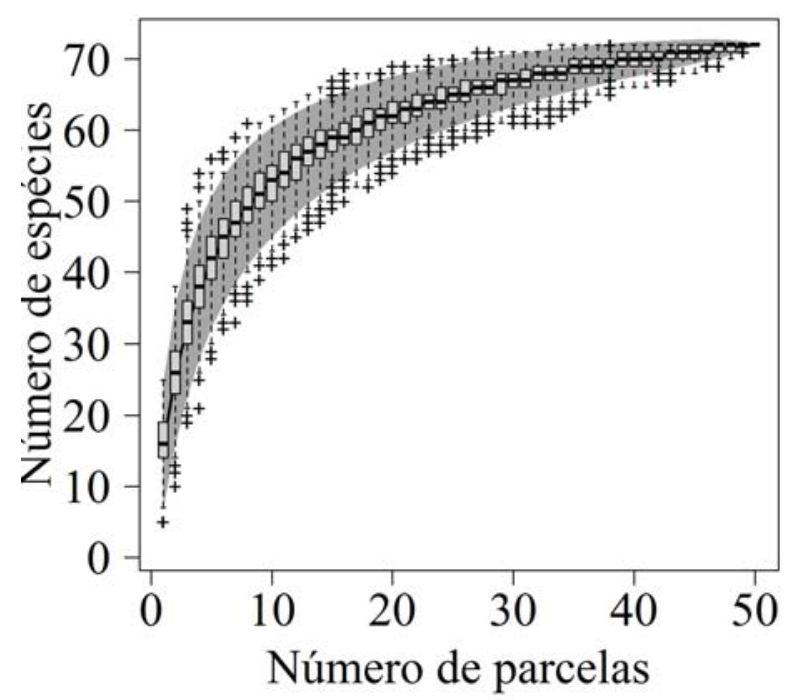

Figura 1 - Curva de rarefação de espécies da comunidade arbórea em fragmentos de Floresta Ombrófila Mista, em Lages, SC.

Figure 1 - Species rarefaction curve of tree community in Araucaria Forest fragments, in the municipality of Lages, SC. apresentando variações relacionadas aos fragmentos analisados (Figura 2). De fato, o teste NPMANOVA demonstrou diferença significativa $(p=0,001)$ na estrutura da comunidade arbórea entre os fragmentos. Os autovalores dos eixos 1 e 2 da DCA foram, respectivamente, de 0,2154 e 0,1718 , podendo ser considerados baixos.

A análise de espécies indicadoras demonstrou a existência de diferenças florístico-estruturais entre os fragmentos, apontando espécies com associações significativas com fragmentos 1, 2 e 3 (Tabela 2). As espécies com os maiores valores indicadores nesses três fragmentos foram Calyptranthes conccina (fragmento 1), Dalbergia frutescens (Vell.) Britton (fragmento 2) e Podocarpus lambertii (fragmento 3). O fragmento 4 não apresentou nenhuma espécie indicadora.

\section{DISCUSSÃO}

A riqueza (73 espécies) e a diversidade ( $\mathrm{H}^{\prime}=$ $3,6)$ encontradas podem ser consideradas elevadas, enquanto a dominância ecológica $(J=0,86)$ pode ser

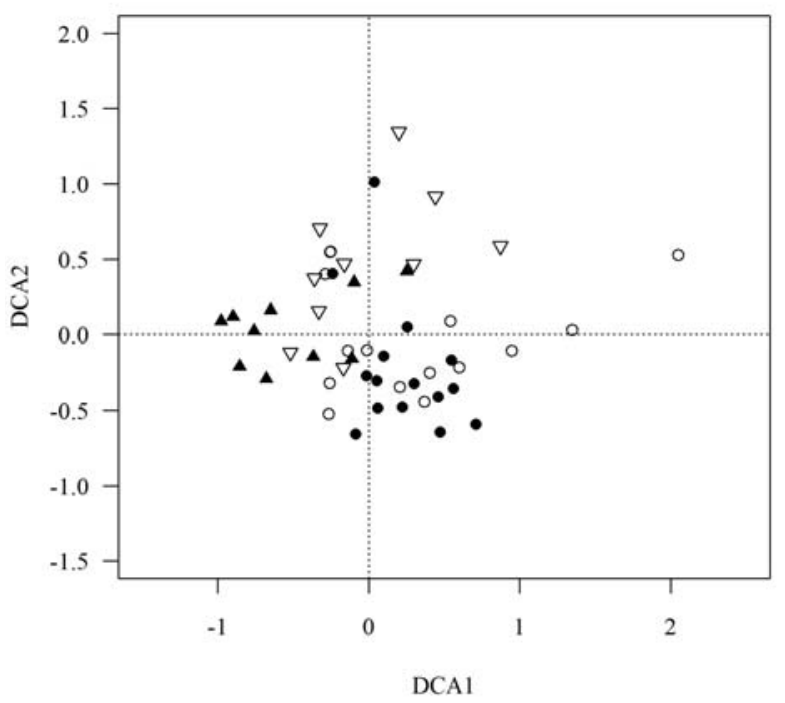

Figura 2 - Análise de Correspondência Retificada (DCA) com parcelas do fragmento $1(\bullet)$, fragmento $2(\nabla)$, fragmento $3(\boldsymbol{\Delta})$ e fragmento $4(\bigcirc)$ em uma área de Floresta Ombrófila Mista, em Lages, SC.

Figure 2 - Detrended correspondence analysis (DCA) with parcels of fragment $1(\bullet)$, fragment $2(\nabla)$, fragment $3(\boldsymbol{\Delta})$ and fragment $4(\bigcirc)$ in an Araucaria Forest area, in the municipality of Lages, SC.

Revista Árvore, Viçosa-MG, v.38, n.5, p.779-786, 2014 
Tabela 2 - Espécies indicadoras de um sistema de quatro fragmentos de Floresta Ombrófila Mista no Município de Lages, SC.

Table 2 - Indicator species of a system of four Araucaria Forest fragments, in the municipality of Lages, SC.

\begin{tabular}{|c|c|c|c|}
\hline Fragmentos & Espécies & Valor indicador & $p$ \\
\hline \multirow[t]{5}{*}{1} & Calyptranthes concinna & 0,5706 & 0,001 \\
\hline & Myrcia palustris & 0,4615 & 0,001 \\
\hline & Drimys brasiliensis & 0,3921 & 0,012 \\
\hline & Jacaranda puberula & 0,3386 & 0,042 \\
\hline & Myrciaria delicatula & 0,3380 & 0,023 \\
\hline \multirow[t]{6}{*}{2} & Dalbergia frutescens & 0,4961 & 0,002 \\
\hline & Xylosma ciliatifolia & 0,4865 & 0,002 \\
\hline & Cinnamodendron dinisii & 0,3219 & 0,045 \\
\hline & Dasyphyllum tomentosum & 0,3088 & 0,032 \\
\hline & Roupala montana & 0,3000 & 0,012 \\
\hline & Casearia obliqua & 0,2743 & 0,039 \\
\hline \multirow[t]{7}{*}{3} & Podocarpus lambertii & 0,7746 & 0,001 \\
\hline & Dasyphyllum spinescens & 0,4500 & 0,004 \\
\hline & Scutia buxifolia & 0,4335 & 0,005 \\
\hline & Zanthoxylum kleinii & 0,4145 & 0,005 \\
\hline & Myrsine coriacea & 0,3424 & 0,029 \\
\hline & Inga lentiscifolia & 0,3000 & 0,017 \\
\hline & Allophylus guaraniticus & 0,2368 & 0,025 \\
\hline 4 & Não apresentou espécies indicadoras & - & - \\
\hline
\end{tabular}

considerada baixa. Outros estudos na região, como de Klauberg et al. (2010) e Nascimento et al. (2011), com o mesmo critério de inclusão de indivíduos, encontraram valores baixos de riqueza e diversidade e altos de dominância. Como neste trabalho, a família Myrtaceae também se destacou nos estudos acima e no de Higuchi et al. (2012b), demonstrando a importância dessa família na definição da composição florística de fragmentos de Floresta Ombrófila Mista na região.

A estrutura horizontal dos fragmentos, que se destacou pela elevada participação da Araucaria angustifolia, Matayba elaeagnoides, Sebastiania commersoniana, Podocarpus lambertii e Calyptranthes concinna, confirma o padrão encontrado na região, pois essas espécies também estão entre as mais importantes em estudos realizados em outras áreas de Floresta com Araucária (NASCIMENTO et al., 2001; RONDON-NETO et al., 2002). Isso indica que existe um conjunto de espécies típicas que acompanham Araucaria angustifolia como caracterizadoras da fitofisionomia da FOM.

Também foram, no entanto, observadas espécies (8), Schinus polygamus (Cav.) Cabrera, Nectandra megapotamica (Spreng.) Mez, Strychnos brasiliensis (Spreng.) Mart., Eugenia uniflora L., Myrcia hatschbachii
D. Legrand, Myrcianthes gigantea (D. Legrand) D. Legrand, Myrsine umbellata Mart. e Cupania vernalis Cambess., consideradas como localmente raras. Essas são mais sensíveis a processos de extinção local, devido a eventos ambientais e demográficos estocásticos (BENITEZMALVIDO; MARTÍNEZ-RAMOS, 2003). Dessa forma, considerando o nível de inclusão avaliado (DAP $\geq 5$ $\mathrm{cm}$ ), essas espécies podem ser consideradas entre as mais suscetíveis a desaparecer da área. Em um cenário de extinção local dessas espécies, a recolonização vai depender da existência de um banco de regenerante e, ou, semente e a chegada de propágulos de áreas vizinhas.

A DCA demonstrou organização heterogênea na área, porém, como os autovalores dos eixos 1 e 2 dessa análise foram baixos, os gradientes são curtos e há baixa substituição de espécies entre as parcelas (CARVALHO; FELFILI, 2011). Assim, é possível inferir que a maior parte das espécies tem distribuição ampla, mas apresenta variação na abundância, ocorrendo em maior quantidade somente em algumas parcelas.

A análise de espécies indicadoras reforçou a existência de diferenças florístico-estruturais entre os fragmentos, apontando espécies com associações com fragmentos 1, 2 e 3. No Fragmento 1, das cinco espécies indicadoras, três pertencem à família Myrtaceae, confirmando a elevada

Revista Árvore, Viçosa-MG, v.38, n.5, p.779-786, 2014 
riqueza da família. As três espécies de maior valor de importância na área estudada - Araucaria angustifolia, Matayba elaeagnoides e Sebastiania commersoniana - não foram indicadoras de nenhum fragmento, o que sugere que essas espécies são pouco exigentes em relação à qualidade do hábitat, o que justifica a elevada representatividade delas em todos os fragmentos.

Entre os fatores que podem explicar as variações encontradas entre os fragmentos, destacam-se a estrutura da paisagem, como o isolamento do fragmento (DISLICH et al., 2001), os fatores edáficos (MACHADO et al., 2004; SILVA et al., 2009) e as diferenças no estágio sucessional (DURIGAN et al., 2008). A existência de um histórico de perturbação diferenciado também é importante fator que pode determinar variações estruturais em comunidades arbóreas. No campo foi observada a presença heterogênea de taquarais e bambuzais, que são indicadores de ambientes degradados (SCHMIDT, 2008), principalmente no fragmento 4. A presença dessa forma de vida no interior do fragmento desempenha importante influência sobre o padrão de regeneração natural em fragmentos florestais no Sul do Brasil (BUDKE et al., 2010), pois pode ocorrer intensa cobertura do solo por matéria orgânica e também diminuição da luz no piso da floresta (LARPKERN et al., 2011), reduzindo, assim, a capacidade dos regenerantes de espécies arbóreas de se estabelecerem (MAROD et al., 1999).

\section{CONCLUSÕES}

O perfil florístico e a estrutura da comunidade foram caracterizados pela elevada riqueza da família Myrtaceae e pela alta representatividade de Araucaria angustifolia. No entanto, a organização estrutural do componente arbóreo nos quatro fragmentos não ocorre de forma homogênea, de forma que existe diferença na estrutura da comunidade, com a existência de espécies indicadoras para os fragmentos 1,2 e 3, de uma área de Floresta Ombrófila Mista, na região do Planalto Sul catarinense. É provável que essas diferenças estejam refletindo a heterogeneidade ambiental entre os fragmentos e que pode estar associada a diferentes históricos de perturbação.

\section{AGRADECIMENTO}

Ao CNPq, pela concessão de bolsa em produtividade em pesquisa para o segundo autor.

\section{REFERÊNCIAS}

ANDERSON, M. J. A new method for nonparametric multivariate analysis of variance. Austral Ecology, v.26, n.1, p.23-42, 2001.

ANGIOSPERM PHYLOGENY GROUP. An update of the Angiosperm Phylogeny Group classification for the orders and families of flowering plants: APG III. Botanical Journal of the Linnean Society, v.161, n.2, p.105-121, 2009.

BENITEZ-MALVIDO, J.; MARTÍNEZ-RAMOS, M. Impact of Forest fragmentation on understory plant richness in Amazonia. Conservation Biology, v.17, n.2, p.389-400, 2003.

BUDKE, J. C. et al. Bamboo dieback and tree regeneration responses in a subtropical forest of South America. Forest Ecology and Management, v.260, n.8, p.1345-1349, 2010.

CARVALHO, F. A.; FELFILI, J. M. Variações temporais na comunidade arbórea de uma floresta decidual sobre afloramentos calcários no Brasil Central: composição, estrutura e diversidade florística. Acta Botanica Brasilica, v.25, n.1, p.203-214, 2011.

DISLICH, R. et al. Análise da estrutura de fragmentos florestais no Planalto Paulistano - SP. Revista Brasileira de Botânica, v.24, n.3, p.321-332, 2001.

DUFRÊNE, M.; LEGENDRE, P. Species assemblages and indicator species: the need for a flexible asymmetrical approach. Ecological Monograph, v.67, n.3, p.345-366, 1997.

DURIGAN, G. et al. Estádios sucessionais e fatores geográficos como determinantes da similaridade florística entre comunidades no Planalto Atlântico, Estado de São Paulo, Brasil. Acta Botanica Brasilica, v.22, n.1, p.51-62, 2008.

EMPRESA BRASILEIRA DEPESQUISA AGROPECUÁRIA - EMBRAPA. Solos do Estado de Santa Catarina. Rio de Janeiro: Embrapa Solos, 2004. 727p.

HIGUCHI, P. et al. Influência de variáveis ambientais sobre o padrão estrutural e florístico do componente arbóreo em um fragmento de Floresta Ombrófila Mista Montana, em Lages, SC. Ciência Florestal, v.22, n.1, p.79-90, $2012 \mathrm{a}$. 
HIGUCHI, P. et al. Floristic composition and phytogeography of the tree component of Araucaria Forest fragments in southern Brazil. Brazilian Journal of Botany, v.35, n.2, p.145-157, 2012b.

HIGUCHI, P. et al. Florística e estrutura do componente arbóreo e análise ambiental de um fragmento de Floresta Ombrófila Mista Altomontana no município de Painel, SC. Ciência Florestal, v.23, n.1, p.153-164, 2013.

\section{INSTITUTO BRASILEIRO DE GEOGRAFIA E} ESTASTICA - IBGE. Mapa Brasil Climas Escala 1:5.000.000, IBGE, 1978, com adaptações. 2002. Disponível em ftp:// geoftp.ibge.gov.br/mapas_tematicos/mapas_murais/ clima.pdf. Acessado em 23 de maio 2014.

\section{INSTITUTOBRASILEIRODE GEOGRAFIAE} ESTASTICA - IBGE. Manual técnico da vegetação brasileira. Rio de Janeiro: 2012. 271p.

KLAUBERG, C. et al. Florística e estrutura de um fragmento de Floresta Ombrófila Mista no Planalto Catarinense. Biotemas, v.23, n.1, p.35-47, 2010.

LARPKERN, P. et al. Bamboo dominance reduces tree regeneration in a disturbed tropical forest. Oecologia, v.165, n.1, p.165-168, 2011.

MACHADO, E. M. et al. Análise comparativa da estrutura e flora do compartimento arbóreoarbustivo de um remanescente florestal na Fazenda Beira-Lago, Lavras, MG. Revista Árvore, v.28, n.4, p.499-516, 2004.

MAROD, D. et al. Structural dynamics of a natural mixed deciduous forest in western Thailand.

Journal of Vegetation Science, v.10, n.6, p.777-786, 1999.

MUELLER-DOMBOIS, D.; ELLENBERG, H. Aims and of vegetation ecology. New York: John Wiley \& Sons, 1974. 547p.

NASCIMENTO, A. R. T. et al. Estrutura e padrões de distribuição espacial de espécies arbóreas em uma amostra de Floresta Ombrófila Mista em Nova Prata, RS. Ciência Florestal, v.11, n.1, p.105-119, 2001.

NASCIMENTO, A. R. T. et al. Estrutura e classificação de um remanescente de floresta ripária no município de Lages, SC. Ciência Florestal, v.21, n.2, p.209-218, 2011.

NEGRINI, M. et al. Dispersão, distribuição espacial e estratificação vertical da comunidade arbórea em um fragmento florestal no Planalto Catarinense.

Revista Árvore, v.36, n.5, p.919-930, 2012.

OKSANEN, J. et al. Vegan: Community Ecology Package. R package version 1.17-2. Disponível em: $<$ http://CRAN.R-project.org/package=vegan $>$ Acesso em: 15 dez. de 2012.

R DEVELOPMENT CORE TEAM. R: A language and environment for statistical computing. R Foundation for Statistical Computing, 2012. Disponível em: <http://www.R-project.org> Acesso em: 15 dez. de 2012.

ROBERTS, D. W. Labdsv: Ordination and multivariate analysis for ecology. R package version 1.4-1. 2012. Disponível em: <http:// CRAN.R-project.org/package=labdsv $>$ Acesso em: 15 dez. de 2012.

RONDON-NETO, R. M. et al. Análise florística e estrutural de um fragmento de Floresta Ombrófila Mista Montana, situado em Criúva, RS - Brasil. Ciência Florestal, v.12, n.1, p.29-37, 2002.

SCHMIDT, R. A tribo Bambuseae Nees (Poaceae, Bambusoideae) no Rio Grande do Sul, Brasil. 2008. 137f. Dissertação (Mestrado em Botânica) Universidade Federal do Rio Grande do Sul, Porto Alegre, 2008.

SILVA, A. C. et al. Florística e estrutura da comunidade arbórea em fragmentos de floresta aluvial em São Sebastião da Bela Vista, Minas Gerais, Brasil. Revista Brasileira de Botânica, v.32, n.9, p.283-297, 2009.

SILVA, A. C. et al. Relações florísticas e fitossociologia de uma Floresta Ombrófila Mista Montana secundária em Lages, Santa Catarina. Ciência Florestal, v.22, n.1, p.193-206, 2012.

SILVA, A. C. et al. Caracterização fitossociológica e fitogeográfica de um trecho de floresta ciliar em Alfredo Wagner, SC, como subsídio para restauração ecológica. Ciência Florestal, v.23, n.4, p.579-593, 2013. 